\title{
Detection of $\mathrm{OH}$ in an atmospheric flame at $1.5 \mu \mathrm{m}$ using optical frequency comb spectroscopy
}

\author{
Lucile Rutkowski, ${ }^{1}$ Alexandra C. Johansson, ${ }^{1}$ Damir Valiev, ${ }^{2}$ Amir Khodabakhsh,,${ }^{1}$ Arkadiusz Tkacz, ${ }^{3}$ Florian M \\ Schmidt, ${ }^{2}$ and Aleksandra Foltynowicz ${ }^{* 1}$ \\ ${ }^{1}$ Department of Physics, Umeå University, 90187 Umeå, Sweden, \\ ${ }^{2}$ Thermochemical Energy Conversion Laboratory, Department of Applied Physics and Electronics, Umeå \\ University, 90187 Umeå, Sweden \\ ${ }^{3}$ Military University of Technology, Institute of Optoelectronics, Kaliskiego 2, 00-908 Warsaw, Poland
}

Received November 15, 2016; accepted December 23, 2016; published December 31, 2016

\begin{abstract}
We report broadband detection of $\mathrm{OH}$ in a premixed $\mathrm{CH}_{4}$ /air flat flame at atmospheric pressure using cavity-enhanced absorption spectroscopy based on an Er:fiber femtosecond laser and a Fourier transform spectrometer. By taking ratios of spectra measured at different heights above the burner we separate twenty $\mathrm{OH}$ transitions from the largely overlapping water background. We retrieve from fits to the $\mathrm{OH}$ lines the relative variation of $\mathrm{OH}$ concentration and flame temperature with a height above the burner and compare them with the 1D simulations of flame structure.
\end{abstract}

Absorption spectroscopy enables calibration-free detection of intermediate combustion products, such as the $\mathrm{OH}$ radical, which is important for the understanding and modelling of flame chemistry. The $\mathrm{OH}$ radical has been detected using cavity ring-down spectroscopy (CRDS) in the ultraviolet spectral range [1], both in low pressure [2] and atmospheric [3] flames. However, performing $\mathrm{OH}$ detection in the telecom near-infrared (NIR) wavelength range is more attractive because of the availability of laser sources and optical components. Until now, various techniques have been used for $\mathrm{OH}$ detection in flames around $1.5 \mu \mathrm{m}$, such as CRDS [4], cavityenhanced absorption spectroscopy [5], wavelength modulation spectroscopy (WMS) [6] and intra-cavity laser absorption spectroscopy [7]. One of the main problems with $\mathrm{OH}$ detection at this wavelength is the strong interference of water absorption lines. The selectivity can be increased by lowering the flame pressure, which decreases the pressure broadening and spectral overlap [4, 7]. However, many industrial applications require measurements under atmospheric or higher pressures [8]. The lack of accurate parameters of high-temperature water transitions around $1.5 \mu \mathrm{m}$ prevents unambiguous identification of the water lines [9, 10]. A way to circumvent this problem has been to measure NIR water spectra at different temperatures and subtract them from the spectra measured in the flame [5, 6]. This, however, often requires extrapolation towards the flame

${ }^{*}$ E-mail: aleksandra.foltynowicz@umu.se temperature and introduces uncertainties and distortion in the $\mathrm{OH}$ spectra.

Here we use NIR cavity-enhanced optical frequency comb spectroscopy to detect broadband $\mathrm{OH}$ spectra in a premixed $\mathrm{CH}_{4}$ /air flat flame of a stabilized burner at atmospheric pressure without the interference of water. We isolate the $\mathrm{OH}$ lines from the water background by taking ratios of spectra measured at different heights above the burner (HABs). This works because water is a final combustion product and therefore its concentration is almost constant above the production zone. We retrieve from our spectra the relative variation of the $\mathrm{OH}$ concentration and flame temperature with $\mathrm{HAB}$ and compare them to a 1-D model of the flame.

The experimental setup is an improved version of that described in [10]. It is based on an optical frequency comb generated by an Er:fiber femtosecond laser, a $60 \mathrm{~cm}$ long open-air cavity containing the flame with a $3.8 \mathrm{~cm}$ diameter, and a fast-scanning Fourier transform spectrometer (FTS). The comb is locked to the cavity using the two-point Pound-Drever-Hall technique [11]. The cavity finesse has been lowered from 1000 to 150 to decrease absorption sensitivity in order to reduce the number of water transitions involved in spectral analysis. The burner is fixed on a vertical translation stage that allows tuning of the $\mathrm{HAB}$ and is operated at the stoichiometric fuel/air ratio with a total flow rate of 10 $\mathrm{L} / \mathrm{min}$. The flame has already been experimentally characterized in terms of water concentration and temperature using WMS [12]. The 1D calculations of chemical structure of the flame were performed in Cantera using the GRI Mech 3.0 chemical kinetics mechanism.

An aluminium shield is placed around the burner to prevent the previously observed misalignment caused by the heat expansion of a breadboard holding the cavity. Secondly, the collimator collecting the cavity transmitted light into a fiber leading to the FTS is now placed directly after the output mirror to reduce the influence of the beam pointing instability. Thirdly, the output mirror is realigned after turning on the flame and at each $\mathrm{HAB}$ to compensate 
for the beam steering induced by the temperature gradient in the flame. These improvements reduced the intensity drop by an order of magnitude and intensity fluctuations by a factor of 2 compared to the previous system, allowing better baseline quantification.

The FTS acquires the spectrum spanning $120 \mathrm{~cm}^{-1}$ with a resolution of $0.033 \mathrm{~cm}^{-1}$ in $0.4 \mathrm{~s}$, and each measurement is averaged 20 times. High-temperature spectra are normalized against the background measured at room temperature when the flame is off. The baseline is corrected for etalons using low frequency sine functions and multiplied by 1.02 to compensate for the residual intensity drop caused by pointing instability.

Figure 1(a) shows spectra measured in the flame at HAB of $2.5,3.5$ and $5 \mathrm{~mm}$. They contain thousands of overlapping lines, where a large majority is identified as hot water transitions by comparing to a new water line list [13], and 20 lines as $\mathrm{OH}$ transitions using line positions from the HITRAN database [14]. A spectral range containing two of the $\mathrm{OH}$ lines is enlarged in Fig. 1(b). Because of the strong overlap with water and the lack of reliable high-temperature water line parameters in this spectral range it is nearly impossible to extract quantitative information from the $\mathrm{OH}$ absorption lines. However, the simulations, shown in Fig. 1(c), predict that above $\mathrm{HAB}$ of $2.5 \mathrm{~mm}$ both water concentration and flame temperature are relatively constant, while the $\mathrm{OH}$ concentration decreases. This is observed in our spectra, where the $\mathrm{OH}$ line intensities change with $\mathrm{HAB}$, while the water line intensities remain constant. Therefore dividing the spectra measured at different HABs by a reference spectrum measured at $\mathrm{HAB}$ of $2.5 \mathrm{~mm}$ cancels water lines to a large extent and reveals the $\mathrm{OH}$ transitions, as shown in Fig. 2 for a ratio of spectra taken at HABs of 5 and $2.5 \mathrm{~mm}$. The ten $\mathrm{OH}$ doublets belong to four absorption bands identified in the figure. The lines above $6400 \mathrm{~cm}^{-1}$ and the PP11.5ff line of the ${ }^{2} \mathrm{X}_{1 / 2} \leftarrow{ }^{0} \mathrm{X}_{1 / 2}$ band are slightly distorted by the residual structure on the baseline that originates from the incomplete cancellation of water lines. However, all other lines (underlined in the figure) are free from distortion and can be used for concentration and temperature retrieval by spectral fitting.

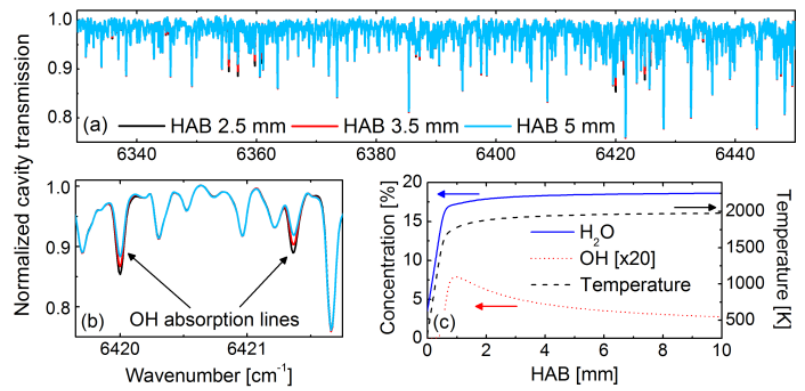

Fig. 1. (a) Normalized cavity transmission measured at different heights above the burner (HABs). (b) Enlargement of a spectral range containing two of the $\mathrm{OH}$ absorption lines. (c) Simulations of water and $\mathrm{OH}$ concentrations and flame temperature as a function of $\mathrm{HAB}$.

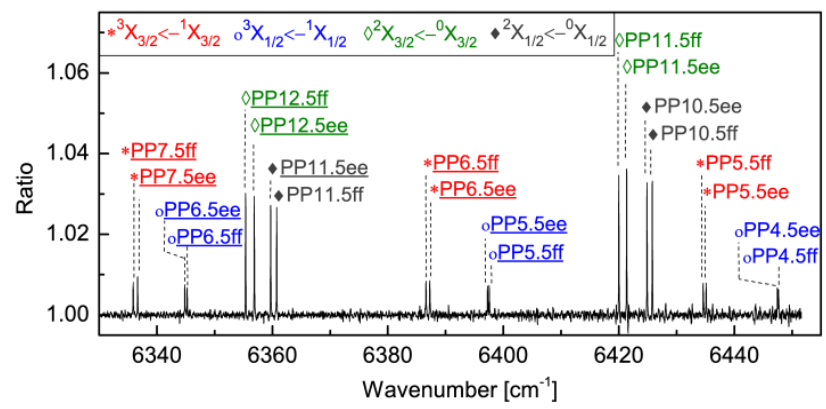

Fig. 2. OH spectrum obtained by taking the ratio of spectra measured at HABs of 5 and $2.5 \mathrm{~mm}$ with transitions belonging to 4 absorption bands. The underlined transitions are used for retrieval of $\mathrm{OH}$ concentration and flame temperature.

We use the three lines of the ${ }^{2} \mathrm{X}_{3 / 2} \leftarrow{ }^{0} \mathrm{X}_{3 / 2}(\diamond$, green) and ${ }^{2} \mathrm{X}_{1 / 2} \leftarrow{ }^{0} \mathrm{X}_{1 / 2}$ (•, grey) bands around $6360 \mathrm{~cm}^{-1}$ (underlined in Fig. 2) to determine the $\mathrm{OH}$ concentration as they have a weak temperature sensitivity of $-7.5 \times 10^{-5}$ $\mathrm{K}^{-1}$ around 1900K [14]. The four doublets of the ${ }^{3} \mathrm{X}_{3 / 2} \leftarrow$ ${ }^{1} \mathrm{X}_{3 / 2}$ (*, red) and ${ }^{3} \mathrm{X}_{1 / 2} \leftarrow{ }^{1} \mathrm{X}_{1 / 2}$ (॰, blue, both underlined) bands have a stronger temperature sensitivity, equal to $5.4 \times 10^{-4} \mathrm{~K}^{-1}$ at $1900 \mathrm{~K}$, and are therefore used to determine the temperature. Figure 3(a) shows the spectral range containing three lines used for concentration retrieval together with fits, made in small ranges around each line, and residuals. The fitted model is based on the ratio of two cavity transmission functions [11] and the $\mathrm{OH}$ absorption lines are calculated using Voigt profiles. The $\mathrm{OH}$ line positions, lower-state energies and the total partition function are taken from the HITRAN database [14]. Note that the line intensities in these spectra do not depend on room-temperature linestrengths, since these cancel when two spectra are divided. The $\mathrm{OH}$ concentration $(0.28 \%)$ and flame temperature $(1900 \mathrm{~K})$ at the reference $\mathrm{HAB}$ are fixed to the values from the 1-D simulations. The temperature at the higher HAB is also fixed to $1900 \mathrm{~K}$ because of the weak temperature sensitivity of the linestrengths. The fitting parameters are
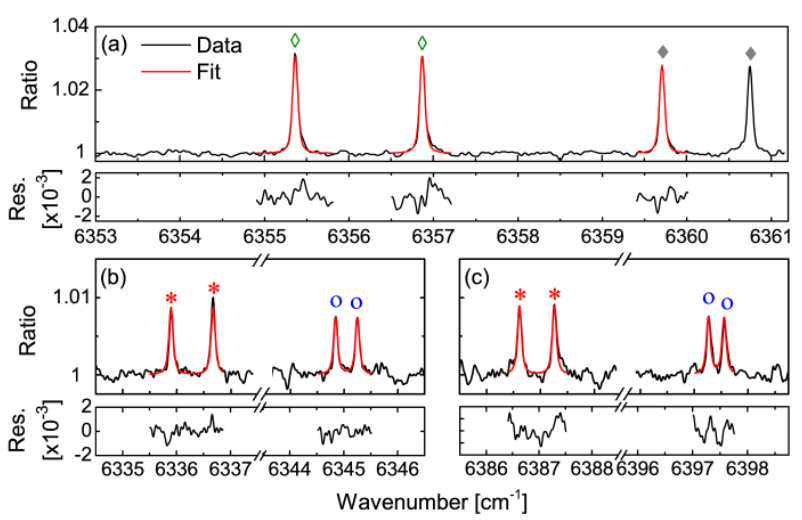

Fig. 3. OH lines with fits for (a) concentration and (b)-(c) temperature retrieval together with residuals (lower panels).

the concentration at the higher $\mathrm{HAB}$ and the roomtemperature linewidth (assumed equal for all lines), where 
the temperature coefficient from HITRAN is used to calculate the high-temperature linewidth. The retrieved room-temperature linewidth is $0.068(2) \mathrm{cm}^{-1}$, except for the two lower HABs, where it is larger $\left[0.084(3) \mathrm{cm}^{-1}\right.$ and $0.072(2) \mathrm{cm}^{-1}$ at 3 and $3.5 \mathrm{~mm}$, respectively], most probably because of the influence of the cavity dispersion, which is neglected here. The concentrations retrieved from these fits are used as input parameters in fits to the four doublets used for temperature determination, shown in Fig. 3(b-c). Here the fitted parameters are the roomtemperature linewidth and the flame temperature at the higher HAB. The linewidth is assumed to be the same for the 8 lines and equal to the linewidth at the reference temperature. The retrieved linewidth is $0.112(3) \mathrm{cm}^{-1}$, apart from the HABs of 3 and $3.5 \mathrm{~mm}$ where it is found equal to $0.16(1) \mathrm{cm}^{-1}$ and $0.120(5) \mathrm{cm}^{-1}$, respectively.

The $\mathrm{OH}$ concentrations (red markers) and flame temperature (black markers) obtained from the fits are compared to simulations (solid and dashed curves) in Fig. 4. The error bars on the concentration values (roughly the size of the markers) take into account the statistical error from the fit $(0.5 \%)$ and the uncertainty induced by the neglected temperature sensitivity of the ${ }^{2} \mathrm{X}_{3 / 2} \leftarrow{ }^{0} \mathrm{X}_{3 / 2}$ and ${ }^{2} \mathrm{X}_{1 / 2} \leftarrow{ }^{0} \mathrm{X}_{1 / 2}$ bands $(0.4 \%)$. The uncertainty of the temperature measurement is given by the fit error $(0.5 \%)$ and by the concentration uncertainty, which translates into a temperature error via the temperature sensitivity of the ${ }^{3} \mathrm{X}_{3 / 2} \leftarrow{ }^{1} \mathrm{X}_{3 / 2}$ and ${ }^{3} \mathrm{X}_{1 / 2} \leftarrow{ }^{1} \mathrm{X}_{1 / 2}$ bands (1\%). The uncertainty on the cavity finesse measurement of $5 \%$ is not included in the error bars. The fit results follow the trend predicted by simulations rather well. The drop of $\mathrm{OH}$ concentration at large $\mathrm{HABs}$ can be explained by the decrease of the flame diameter with $\mathrm{HAB}$ due to product gas diffusion and mixing with the surrounding air, which is not taken into account in the 1-D model. The decrease of temperature at the larger $\mathrm{HAB}$ has been observed previously [12] and can be attributed to radiative heat loss (not included in the model).

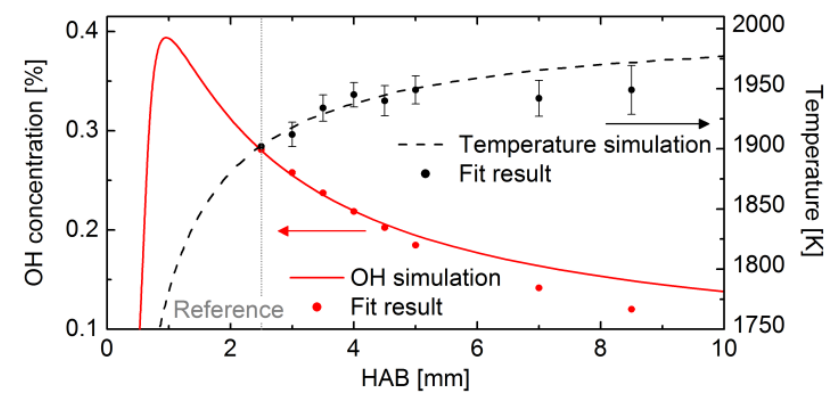

Fig. 4. $\mathrm{OH}$ concentration (in red) and temperature (in black) retrieved from fits to $\mathrm{OH}$ spectra (markers) compared to simulations (curves).

In conclusions, we demonstrated the quantitative detection of $\mathrm{OH}$ in an atmospheric pressure flame using NIR cavity-enhanced optical frequency comb spectroscopy. The broad spectral bandwidth of the comb allows the measurement of twenty $\mathrm{OH}$ transitions from four different bands, the largest number ever detected simultaneously with absorption spectroscopy in this range. We remove water interference by taking ratios of spectra measured at different HABs above the production zone and isolate multiple undistorted $\mathrm{OH}$ lines. We retrieve $\mathrm{OH}$ concentration and flame temperature relative to the reference $\mathrm{HAB}$ by fitting a model to the spectra. The broad spectral bandwidth also yields better immunity to baseline drifts, which can be removed during the fitting process. The relative $\mathrm{OH}$ concentration and flame temperature obtained from the fits compare well to 1-D simulations. The retrieval of absolute flame parameters will require simultaneous fitting of $\mathrm{OH}$ and water transitions at each $\mathrm{HAB}$, which in turns relies on the availability of an accurate model of high-temperature water spectra. The calculations of a new water line list are under way and the broadband high-temperature spectra measured in the flame with cavity-enhanced optical frequency comb spectroscopy will allow its verification [13].

We acknowledge financial support from the Swedish Research Council (621-2012-3650), the Swedish Foundation for Strategic Research (ICA12-0031), the Swedish Energy Agency (36160-1) and the Swedish strategic research program Bio4Energy. The authors thank Yuwei Jin for early optimization of the setup.

\section{References}

[1] G. Meijer, M.G. Boogaarts, R.T. Jongma, D.H. Parker, A.M. Wodtke, Chem. Phys. Lett. 217, 112 (1994).

[2] S. Cheskis, I. Derzy, V.A. Lozovsky, A. Kachanov, D. Romanini, Appl. Phys. B 66, 377 (1998).

[3] X. Mercier, E. Therssen, J.F. Pauwels, P. Desgroux, Chem. Phys. Lett. 299, 75 (1999).

[4] J. Scherer, D. Voelkel, D. Rakestraw, Appl. Phys. B 64, 699 (1997).

[5] R. Peeters, G. Berden and G. Meijer, Appl. Phys. B 73, 65 (2001).

[6] T. Aizawa, Appl. Opt. 40, 4894 (2001).

[7] B. Löhden, S. Kuznetsova, K. Sengstock, V. M. Baev, et al., Appl. Phys. B 102, 331 (2011).

[8] A. Matynia, M. Idir, J. Molet, C. Roche, et al., Appl. Phys. B 108, 393 (2012).

[9] R.S. Watt, T. Laurila, C.F. Kaminski, J. Hult, Appl. Spectrosc. 63, 1389 (2009).

[10] C. Abd Alrahman, A. Khodabakhsh, F.M. Schmidt, Z. Qu, A. Foltynowicz, Opt. Expr. 22, 13889 (2014).

[11] A. Foltynowicz, P. Masłowski, A.J. Fleisher, B.J. Bjork, J. Ye, Appl. Phys. B 110, 163 (2013).

[12] Z. Qu, R. Ghorbani, D. Valiev, F. M. Schmidt, Opt. Expr. 23, 16492 (2015).

[13] L. Rutkowski, A. Khodabakhsh, A.C. Johansson, D.M. Valiev, et al., CLEO: Science and Innovations DOI SW4H.8 (2016).

[14] L.S. Rothman, I.E. Gordon, Y. Babikov, A. Barbe, et al., J. Quant. Spectrosc. Radiat. Transf. 130, 4 (2013). 